\title{
Editorial: Genetics of Complex Traits and Diseases From Under-Represented Populations
}

\author{
Segun Fatumo ${ }^{1,2 *}$, Tinashe Chikowore ${ }^{3,4}$ and Karoline Kuchenbaecker ${ }^{5,6}$ \\ ${ }^{1}$ The African Computational Genomics (TACG) Research Group, MRC/UVRI and LSHTM, Entebbe, Uganda, ${ }^{2}$ The Department of \\ Non-communicable Disease Epidemiology, London School of Hygiene and Tropical Medicine, London, United Kingdom, \\ ${ }^{3}$ Sydney Brenner Institute for Molecular Bioscience, Faculty of Health Sciences, University of the Witwatersrand, Johannesburg, \\ South Africa, ${ }^{4}$ MRC/Wits Developmental Pathways for Health Research Unit, Department of Paediatrics, Faculty of Health \\ Sciences, University of the Witwatersrand, Johannesburg, South Africa, ${ }^{5}$ Division of Psychiatry, University College of London, \\ London, United Kingdom, ${ }^{6}$ UCL Genetics Institute, University College London, London, United Kingdom
}

Keywords: genomic, underrepresentation, GWAS, genome-wide association study, genetic, complex disease

\section{Editorial on the Research Topic}

\section{Genetics of Complex Traits and Diseases From Under-Represented Populations}

The Inclusion of ethnically diverse populations in genetic research can provide deep insights into specific pathogenic variants that differ across and within populations. Such insights may be useful to guide diagnosis and provide relevant clinical interventions (Gurdasani et al., 2019; Chikowore et al., 2021; Fatumo et al., 2021a; Fatumo et al., 2021b). Unfortunately, the vast majority of the genetic studies have been performed in European ancestry populations and few in Africa (Kuchenbaecker et al., 2019; Chikowore et al., 2021).

We accepted only high-quality research work in this research topic, including review manuscripts

Edited and reviewed by: Jordi Pérez-Tur

Institute of Biomedicine of Valencia

(CSIC), Spain

*Correspondence: Segun Fatumo

segun.fatumo@/shtm.ac.uk

Specialty section: This article was submitted to Genetics of Common and Rare Diseases, a section of the journal Frontiers in Genetics

Received: 18 November 2021 Accepted: 21 December 2021 Published: 17 January 2022

Citation:

Fatumo S, Chikowore $T$ and Kuchenbaecker K (2022) Editorial: Genetics of Complex Traits and Diseases From Under-

Represented Populations.

Front. Genet. 12:817683. doi: $10.3389 /$ fgene.2021.817683 describing the genetics of diseases in underrepresented populations such as African, Asian, Latin American, and other indigenous populations. Topics could include Genome-wide association studies, Gene-environment interactions, Population genetics, epigenetics, pharmacogenomics, Polygenic/Genetic Risk Scores. The final topic issue has 10 published articles out of 18 submitted manuscripts, covering various genetic studies in African, Asian, Arab, and other previously under-studied populations.

Most of the studies demonstrated that better representation of ethnically and ancestrally diverse populations is crucial for our ability to comprehensively characterise the genetic architecture of complex traits and human disease. For example, Font-Porterias et al. analysed genome-wide array data and exome sequences of 89 healthy Spanish Roma individuals to investigate chronic diseases and traits. The Roma represents the largest trans-national minority ethnic group in Europe, originating from South Asia and receiving extensive gene flow from West Eurasia. The authors found frequency differences between the Roma and other non-Roma populations for disease-causing variants and variants linked to drug response. The work demonstrated the value of merging population genetics and genetic epidemiology methodology.

Other papers demonstrated how such differences in clinically relevant genetic variation can impact health inequalities, if populations are not sufficiently represented in genetic research. Chande et al. analysed the effect of genetic ancestry and ethnicity on observed disease prevalence. They predicted disease risk in Colombia populations by mainly comparing the disease prevalence and socioeconomic indicators of Colombia's three major ethnic groups; from Mestizo, Afro-Colombian, and the Indigenous group. The study demonstrated a strong link between ethnicity, ancestry, and health outcomes. In their mini review Nagaraj and Toombs discussed "the history of pharmacogenomics and highlight the inequalities that must be addressed to ensure equal access 
to pharmacogenomic-based healthcare and reviewed the efforts to conduct the pharmacogenomic profiling of chronic diseases among Australian Indigenous populations including the impact of the lack of drug safety-related information on potential ADRs among individuals in these Australian Indigenous communities".

Papers in this special issue actively addressed underrepresentation by investigating the genetics of complex traits in diverse populations. One approach used to address the imbalance is synthesising existing studies to empower more generalizable conclusions. Soremekun et al. meta-analysed summary statistics from genome-wide association studies of multiple African data, carried out a Bayesian finemapping, and assessed the causal relationship between White Blood Cell (WBC) subtypes and Asthma including a two-sample Mendelian Randomization (MR) analysis. This study identified five novel genes not previously reported associated with any WBC subtype. Their MR analysis showed that monocytes count and neutrophils counts are associated with an increased risk of asthma. Singh et al. conducted a systematic review to summarise studies on genetic association studies for blood pressure-related traits in populations with African ancestry. The review highlighted the limited number of available studies, especially in Africa. Nevertheless, across the five blood pressure-related traits, they found that 26 genome-wide significantly associated SNPs have been identified, including 12 associations that have not previously been described in non-African studies.

Similarly, Al-Homedi et al. leveraged several small-scale genome-wide association studies of metabolic syndrome (MetS) performed in Arab populations to investigate the genetic basis of MetS in the population. Their systematic review and meta-analysis identified 36 studies and shows that the most frequently studied genes were FTO and VDR. The study failed to find any unique genetic association between MetS and Arabian populations.

It may be unsurprising that other studies in this Special Issue focussed on cardiometabolic outcomes, given the immense public health burden of cardiovascular disease. Dlamini et al. investigated the associations between genetic variation in CYP17A1 and SERPINA6/A1 and circulating glucocorticoid concentrations in black South African adults. The authors found that a variant rs17090691 with $G$ effect allele at SERPINA6/A1 was associated with increased diastolic blood pressure in all adults at $p$-value of $9.47 \times 10^{-6}$. However, no association was seen between these genetic variants and glucocorticoids or between any variants in CYP17A1 and metabolic outcomes after adjusting for multiple testing.

In Wang et al., the authors recruited over 1,400 Han Chinese patients with coronary artery disease. They conducted linear regression analyses on the genetic variants of mitochondrial DNA (mtDNA) and lipidomic traits in two independent groups where they found three associations. They also explored the associations between genetic variants in mitochondrial and lipids traits and reported two significant associations. The study provided "insights into the lipidomic context of mtDNA variations and highlighted the importance of studying mitochondrial genetic variants related to lipid species".

Two of the studies focused on mental health genetics in African populations. Genetic research could play an important role to decrease stigma and better understand the causes of this highly prevalent, yet poorly understood global health problem. Owalla et al. investigated the effects of stress and the genetic variation within the FK506 binding protein 5 (rs1360780) and glucocorticoid receptor (rs10482605) genes on internalizing disorders (ID) status in a CA-HIV cohort in Uganda. Using logistic regression, the authors assessed the relationships between IDs and recent stress, chronic stress, and the investigated genotypes. They reported no significant association between IDs and rs1360780 and rs10482605 polymorphisms within the FKBP5 and glucocorticoid receptor genes nor observed any gene-environment effect on vulnerability IDs in the Ugandan population, but shows that severe recent stress increases the vulnerability to IDs among CA-HIV. The findings further supported that polymorphisms at genetic loci only contributed minimal to the genetic vulnerability to IDs.

Similarly, Kalungi et al. assessed the relationships between acute stress and Internalizing mental disorders (IMDs), and moderation by chronic stress and SNPs in SLC6A4 and TPH2 in Ugandan populations. The authors observed a "statistically significant association between severe acute stress and IMDs. Acute stress was found to interact with 5-HTTLPR-rs25531S-A-S$A$ haplotype to increase the risk for IMDs among Ugandan $\mathrm{HIV}^{+}$ children and adolescents but no evidence for a combined interaction of acute stress, chronic stress, and 5-HTTLPRrs25531 on IMDs".

Overall, the studies in this Special Issue highlighted the importance of genetic research in diverse global populations. They synthesized existing data to enable conclusions for diverse populations, demonstrate population variation linked to clinically relevant genes, discover novel genetic associations, and investigate the implications for health inequalities.

\section{AUTHOR CONTRIBUTIONS}

All authors listed have made a substantial, direct, and intellectual contribution to the work and approved it for publication.

\section{FUNDING}

$\mathrm{SF}$ is an Intermediate international fellow funded by the Wellcome Trust grant (220740/Z/20/Z) at the MRC/UVRI and LSHTM. TC is an international training fellow supported by the Wellcome Trust grant $(214205 / Z / 18 / Z)$. KK is funded by the European Research Council (ERC) under the European Union's Horizon 2020 research and innovation program (Grant agreement No. 948561).

\section{ACKNOWLEDGMENTS}

The guest editors wish to thank all the authors and reviewers for their valuable contributions to this Research Topic and we hope that this collection of articles will be of interest to the medical and genetics community. 


\section{REFERENCES}

Chikowore, T., Kamiza, A. B., Oduaran, O. H., Machipisa, T., and Fatumo, S. (2021). Non-Communicable Diseases Pandemic and Precision Medicine: Is Africa Ready? EBioMedicine 65, 103260. doi:10.1016/j.ebiom.2021.103260

Fatumo, S., Chikowore, T., Kalyesubula, R., Nsubuga, R. N., Asiki, G., Nashiru, O., et al. (2021a). Discovery and Fine-Mapping of Kidney Function Loci in First Genome-Wide Association Study in Africans. Hum. Mol. Genet. 30, 1559-1568. doi: $10.1093 / \mathrm{hmg} / \mathrm{ddab} 088$

Fatumo, S., Karhunen, V., Chikowore, T., Sounkou, T., Udosen, B., Ezenwa, C., et al. (2021b). Metabolic Traits and Stroke Risk in Individuals of African Ancestry: Mendelian Randomization Analysis. Stroke 52, 2680-2684. doi:10.1161/STROKEAHA.121.034747

Gurdasani, D., Carstensen, T., Fatumo, S., Chen, G., Franklin, C. S., PradoMartinez, J., et al. (2019). Uganda Genome Resource Enables Insights into Population History and Genomic Discovery in Africa. Cell 179 (4), 984-1002. e36. doi:10.1016/j.cell.2019.10.004

Kuchenbaecker, K., Telkar, N., Telkar, N., Reiker, T., Walters, R. G., Lin, K., et al. (2019). The Transferability of Lipid Loci across African, Asian and
European Cohorts. Nat. Commun. 10 (1), 4330. doi:10.1038/s41467-01912026-7

Conflict of Interest: The authors declare that the research was conducted in the absence of any commercial or financial relationships that could be construed as a potential conflict of interest.

Publisher's Note: All claims expressed in this article are solely those of the authors and do not necessarily represent those of their affiliated organizations, or those of the publisher, the editors and the reviewers. Any product that may be evaluated in this article, or claim that may be made by its manufacturer, is not guaranteed or endorsed by the publisher.

Copyright (c) 2022 Fatumo, Chikowore and Kuchenbaecker. This is an open-access article distributed under the terms of the Creative Commons Attribution License (CC $B Y)$. The use, distribution or reproduction in other forums is permitted, provided the original author(s) and the copyright owner(s) are credited and that the original publication in this journal is cited, in accordance with accepted academic practice. No use, distribution or reproduction is permitted which does not comply with these terms. 